\title{
Individuals with mild-to-moderate hip osteoarthritis have lower limb muscle strength and volume deficits
}

\author{
Aderson Loureiro ${ }^{1,2,3}$, Maria Constantinou ${ }^{1,4}$, Laura E. Diamond ${ }^{1,5^{*}}$ (D) Belinda Beck ${ }^{1}$ and Rod Barrett ${ }^{1}$
}

\begin{abstract}
Background: Individuals with advanced hip osteoarthritis $(\mathrm{OA})$ exhibit generalized muscle weakness of the affected limb and so clinical practice guidelines recommend strength training for the management of hip OA. However, the extent and pattern of muscle weakness, including any between-limb asymmetries, in early stages of the disease are unclear. This study compared hip and knee muscle strength and volumes between individuals with mild-to-moderate symptomatic and radiographic hip OA and a healthy control group.

Methods: Nineteen individuals with mild-to-moderate symptomatic and radiographic hip OA ( $n=12$ unilateral; $n=7$ bilateral) and 23 age-matched, healthy controls without radiographic hip OA or hip pain participated. Isometric strength of the hip and knee flexors and extensors, and hip abductors and adductors were measured. Hip and thigh muscle volumes were measured from lower limb magnetic resonance images. A full-factorial, two-way General Linear Model was used to assess differences between groups and between limbs.

Results: Participants in the hip OA group demonstrated significantly lower knee flexor, knee extensor, hip flexor, hip extensor and hip abductor strength compared to controls and had significantly lower volume of the adductor, hamstring and quadriceps groups, and gluteus maximus and gluteus minimus muscles, but not tensor fasciae latae or gluteus medius muscles. There were no between-limb strength differences or volume differences within either group.

Conclusions: Atrophic, bilateral hip and knee muscle weakness is a feature of individuals with mild-to-moderate hip OA. Early interventions to target muscle weakness and prevent the development of strength asymmetries that are characteristic of advanced hip OA appear warranted.
\end{abstract}

Keywords: Atrophy, Weakness, OA, MRI, Isometric

\section{Background}

People with hip osteoarthritis (OA) often experience joint pain, stiffness, reduced joint range of motion, and muscle weakness [1-4]. These deficits can limit performance of activities of daily living and diminish quality of life [5]. Hip OA has no cure, and progression to more advanced disease occurs in many patients. Conservative non-pharmacological interventions focus primarily on alleviating pain and improving function [6-11]. Individuals

\footnotetext{
* Correspondence: I.diamond@griffith.edu.au

${ }^{1}$ Menzies Health Institute Queensland, School of Allied Health Sciences, Griffith University, Gold Coast, QLD 4222, Australia

${ }^{5}$ Centre of Clinical Research Excellence in Spinal Pain, Injury \& Health, School of Health \& Rehabilitation Sciences, The University of Queensland, Brisbane, QLD, Australia

Full list of author information is available at the end of the article
}

with advanced hip OA exhibit generalized muscle weakness of the affected limb [12-19], which is underpinned by a combination of muscle atrophy $[16,18,20-22]$, reduced muscle density [14, 21, 22], and muscle inhibition [22]. Clinical practice guidelines recommend land-based therapeutic exercise for the management of hip OA [23], most notably resistance training, which can reduce pain, stiffness and self-reported disability, and improve strength, physical function and joint range of motion [24, 25]. At present however, there is limited understanding of the extent and pattern of muscle weakness in earlier stages of the disease. If muscle weakness were also found to be a feature of mild-moderate hip OA, then interventions such as resistance training that target muscle weakness and prevent the development of strength asymmetries 
characteristic of advanced hip OA [26] may be warranted in earlier stages of the disease.

Most investigations of muscle properties in hip OA have included individuals in advanced stages of the disease [14, 16, 18, 20-22]. Studies that included patients across the early spectrum of disease severity $[12,27]$ reported lower gluteal muscle volumes in individuals with unilateral hip OA compared to their contralateral side and a group of healthy controls. Deficits in hip abduction and internal rotation strength of the affected leg compared to healthy controls were also noted and suggest that muscle weakness could also be a feature of earlier stages of the disease than previously reported. It therefore remains unclear whether muscle weakness and atrophy that precede advanced stages of the disease extend beyond the abductor muscle group of the affected leg to other prime movers (i.e. quadriceps, hamstrings, adductors) within the most affected leg or the contralateral leg. Evidence of between-limb differences in hip and knee muscle strength and/or muscle volume have been reported in advanced hip OA [12, 22] and following total hip replacement [21]. While Grimaldi et al. [20, 28] reported an absence of asymmetry in the volume of the gluteal, piriformis, and tensor fascia latae muscles in mild hip OA, symmetry of other important hip and knee muscles is yet to be assessed. An improved understanding of whether muscle weakness and atrophy in mild-to-moderate hip OA is generalized or specific to certain muscles or muscle groups in the lower extremity is required to appropriately inform and optimise management programs.

The purpose of this study was to compare hip and knee muscle strength and volumes between individuals with mild-to-moderate symptomatic and radiographic hip OA and a healthy control group. Based on evidence from studies which report muscle weakness and atrophy in knee OA [29], it was hypothesized that individuals with mild-moderate hip OA would similarly exhibit muscle weakness and lower limb muscle atrophy, particularly in their (more) affected limb, compared to healthy age-matched controls.

\section{Methods}

\section{Participants}

Individuals aged 45 to 80 years with symptomatic unilateral or bilateral hip osteoarthritis were recruited from local hospital orthopaedic waiting lists to participate in this case-control study. Healthy controls were recruited through advertising and word-of-mouth. All participants were screened through radiographic examination (anterior-posterior radiographs of the pelvis and hips) and self-reported measures of pain and function (modified Harris Hip Score (HHS) [30]). Unilateral and bilateral hip OA participants were required to have hip pain and/or functional limitations during activities of daily living (HHS $\leq 95 ; 0=$ extreme hip problems, $100=$ no hip problems) and had a KellgrenLawrence (KL) grade [31] for their affected hip(s) of 2 or 3 and/or joint space width (JSW) $\leq 3 \mathrm{~mm}$ ). Unilateral hip OA participants had KL scores of 0 or 1 for their contralateral hip. Healthy controls were required to have no hip pain or functional limitations during activities of daily living $(\mathrm{HHS}>95)$ and had $\mathrm{KL}$ grades $\leq 1$ and $\mathrm{JSW}>3 \mathrm{~mm}$ for both hips. KL scores were determined by a single radiologist in a blinded manner from bilateral weight-bearing radiographs performed in 15 degrees of femoral internal rotation [32]. The same radiologist electronically measured supero-medial, apical and supero-lateral hip JSW [33]. Exclusion criteria for both groups included: (i) previous lower limb or back fracture or surgery; (ii) history of trauma to the hip joint or pelvis region; (iii) other forms of arthritis, diabetes, cardiac or circulatory conditions; and (iv) use of corticosteroid medication. All individuals were able to walk without physical assistance or devices.

An a priori power analysis using hip abduction strength data from Zacharias et al. [27] (hip OA = 0.15(0.09); controls $=0.25(0.10))$ estimated a minimum of 12 participants were required in each group (significance level was set at $\alpha=0.05$ and power at 0.80 (one tail)). Participants were enrolled concurrently in another study [34]. This study was approved by the institutional Human Research Ethics Committee and written informed consent was obtained from the participants prior to participating in the study.

\section{Procedures}

Participants initially attended a laboratory session to assess bilateral isometric strength of the lower extremity muscles. Anthropometric measures including height $(\mathrm{m})$ and body mass $(\mathrm{kg})$ were also taken. Body mass index (BMI) was determined as weight divided by the square of height $\left(\mathrm{kg} / \mathrm{m}^{2}\right)$. Within $48 \mathrm{~h}$ of attending the strength testing session, participants underwent bilateral magnetic resonance imaging (MRI) of their lower extremity in a private radiologic clinic. This study conformed to the STROBE statement for reporting case-control studies [35].

Maximal voluntary isometric hip and knee muscle strength was measured using an isokinetic dynamometer (Biodex System 4, Biodex Medical Systems, USA) using a protocol adapted from Carty et al. [36]. Hip flexor, extensor, adductor and abductor strength were assessed while standing in $0^{\circ}$ of hip flexion and adduction (neutral position), with the knee constrained in $60^{\circ}$ of flexion using a post-surgical orthopaedic knee brace, and the ankle in $5^{\circ}$ of plantar flexion. Participants were allowed to apply a light force against the dynamometer head for the purpose of maintaining balance. Knee flexor and extensor strength tests were performed while seated. Knee flexor strength was assessed at $30^{\circ}$ of knee flexion with the hip in $90^{\circ}$ of flexion and the ankle in $5^{\circ}$ of plantar flexion. Knee extensor strength was assessed at $60^{\circ}$ of knee flexion with the 
hip in $70^{\circ}$ of flexion and the ankle in $5^{\circ}$ of plantar flexion. The order of strength measurements was from hip to knee randomized by limb. Participants performed a 5-s practice trial at $75 \%$ of maximal effort for each exercise, followed by a 60 -s rest and a 5 -s maximal contraction. Prior to each maximal effort trial, participants were instructed to contract as hard as they could for 5-s, with verbal encouragement provided to help maximize effort. The instantaneous peak isometric torque for each exercise was adjusted to account for the torque due to the dynamometer attachment and lower limb segments distal to the joint being tested in accordance with the recommendations of Kellis and Baltzopoulos [37], using body segment parameters estimated from Dempster [38]. Isometric strength at each joint, in each direction, was defined as the peak torque measured normalized to body mass $(\mathrm{Nm} / \mathrm{kg})$.

A 3.0 T MRI whole body scanner (Phillips Ingenia, Phillips Medical, Netherlands), was used to image bilateral lower limbs of all participants. Axial plane scans were performed with participants positioned supine in the scanner using body coil arrays placed superiorly on the limbs with contiguous slices taken from approximately 2 -cm superior to the iliac crest to approximately $2-\mathrm{cm}$ inferior to the proximal tibio-fibula joint. Both lower limbs were scanned simultaneously with $\mathrm{T} 1$ weighted 2-dimensional gradient-recall acquisition in the steady state; slice thickness 10-mm, inter-slice gap 1-mm, flip angle $90^{\circ}$; repetition time $677 \mathrm{msec}$, echo time $6.5 \mathrm{msec}$; field of view $280 \times 500 \times 219 \mathrm{~mm}$; $352 \times 499$-pixel matrix; acquisition time $1 \mathrm{~min} 29 \mathrm{~s}$. Volumes of individual muscles (tensor fasciae latae (TFL), gluteus maximus (GMax), gluteus medius (GMed), gluteus minimus (GMin)) and muscle groups (adductors (i.e. magnus, gracilis, brevis, and longus) (Add), quadriceps (i.e. vastus medialis, vastus intermedius, vastus lateralis, rectus femoris) (Quad), hamstrings (i.e. semimembranosus, semitendinosus, biceps femoris) (Hams)) were then calculated using Mimics software (Materialise N.V., Belgium). The ilopsoas muscle was not assessed as it was only partially visible in the MRI scans obtained. Muscles were segmented on a slice-by-slice basis by a single reader (AL) using the semi-automated lasso tool (Fig. 1a). These data were then combined to create the final 3-dimensional (3D) rendering. The 3D-volume object was wrapped using finest detail of $0.50 \mathrm{~mm}$ and a gap closing distance of $1.00 \mathrm{~mm}$, followed by a smoothing process with a factor of 1.0 and 4 iterations. Finally, the muscle volumes were determined by summing all pertinent pixels within the resultant binary volume (Fig. 1b-c). Individual and group muscle volumes were normalized to body mass $\left(\mathrm{cm}^{3} / \mathrm{kg}\right)$. Reliability of muscle segmentation was assessed following the approach described by Grimaldi et al. [20]. In brief this involved the same investigator (AL) segmenting the same image slices from all muscles for a single randomly selected participant on 2 occasions, approximately 2 weeks apart. Intra-rater reliability, as assessed using the intra-class correlation coefficient (ICC) was high, with ICCs for all muscles in excess of 0.985 .

\section{Statistical analysis}

Shapiro-Wilk tests were used to examine data normality. Demographic and clinical variables were compared between groups using independent t-tests or Pearson's chi-square. A full-factorial, two-way General Linear Model was used to assess the effect of a between subject factor (Group) and a within subject factor (Leg) on muscle strength and volume. A priori contrasts were used to assess differences between limbs within each group. Leg was defined as affected/contralateral for participants with unilateral OA and most affected (on the basis of symptoms)/less affected for participants with bilateral OA. The test limb was randomly selected (left/ right) for control participants. Effect sizes for main group effects were computed using Cohen's d. Statistical analyses were performed using SPSS version 17.0 for Windows (SPSS Inc., Chicago, USA) with significance level set at $p<0.05$.

\section{Results}

There were no differences in age, height, or body mass between the hip OA and control groups. On average, participants in the hip OA group had a higher BMI than participants in the control group $(p<0.01)$ (Table 1$)$.

\section{Lower limb strength}

No group by leg interaction effects were detected for any measure of lower-limb strength. A significant main effect of group was detected for knee flexor, knee extensor, hip flexor, hip extensor, hip abductor strength (Table 2 and Fig. 2a), but not hip adductor strength. No significant strength differences were detected between legs within each group.

\section{Hip and knee muscle volume}

No group by leg interaction effects were detected for any measure of hip or knee muscle volume. A significant main effect of group was detected for GMax, GMin, Add, Hams, and Quad volume (Table 2 and Fig. 2b), but not TFL and GMed. No significant volume differences were detected between legs within each group.

\section{Discussion}

This study compared bilateral isometric hip and knee muscle strength and hip and knee muscle volume between individuals with symptomatic and radiographic mild-to-moderate hip OA and healthy controls. Consistent with our hypothesis, individuals with hip OA tended to be weaker and have less muscle volume than those in 

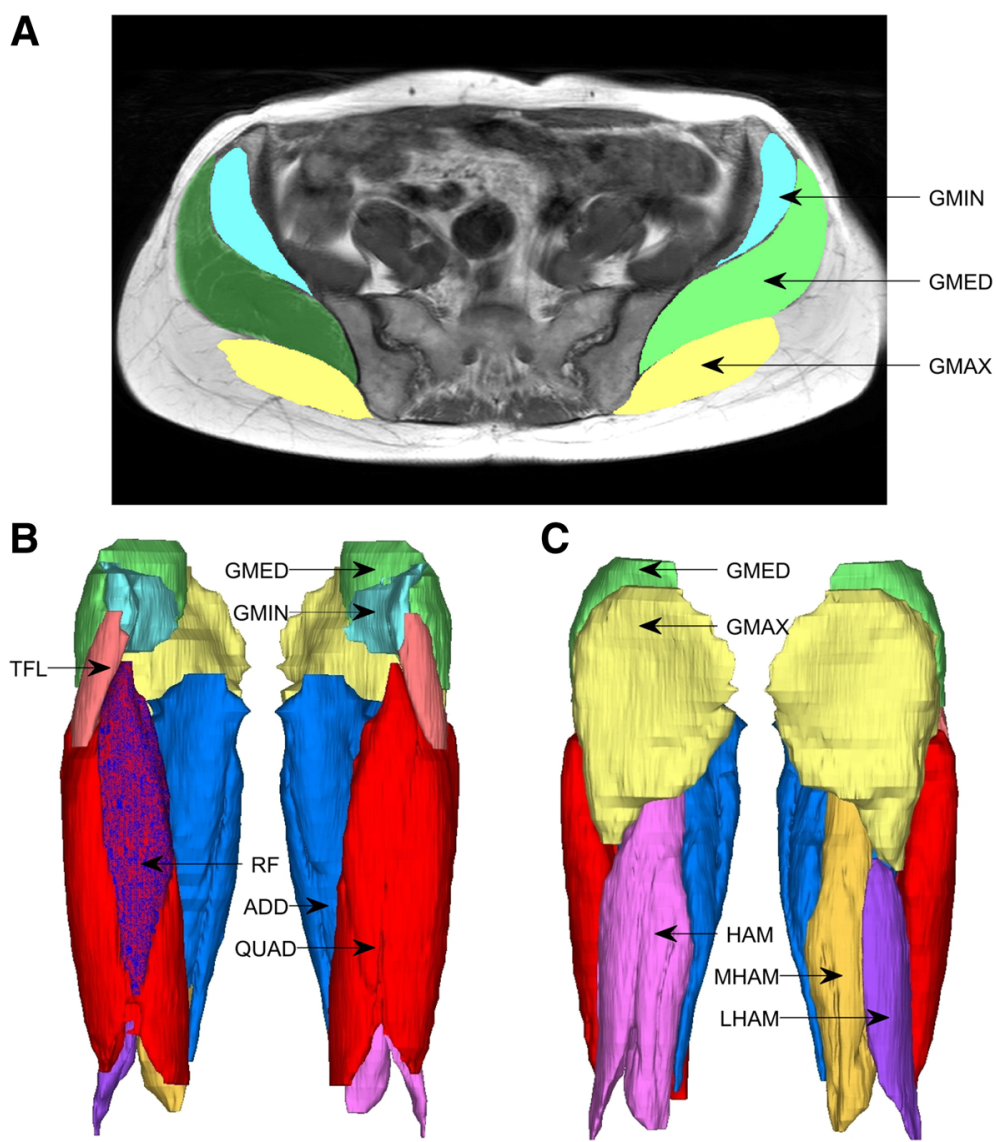

Fig. 1 Muscle and muscle group segmentation from magnetic resonance images of a representative healthy control participant; a superior view of muscle masks segmented from an individual transverse plane slice; b-c anterior and posterior views, respectively, of 3D rendering of thigh and hip muscles (GMIN-gluteus minimus; GMED-gluteus medius; GMAX-gluteus maximus; TFL-tensor fasciae latae; ADD-adductors; QUAD-quadriceps; HAM-hamstrings)

Table 1 Participant characteristics of hip osteoarthritis and control groups

\begin{tabular}{|c|c|c|c|c|c|c|c|c|}
\hline Characteristic & \multicolumn{2}{|c|}{ Unilateral hip OA $n=12$} & \multicolumn{2}{|c|}{ Bilateral hip OA $n=7$} & \multicolumn{2}{|l|}{ Hip OA $n=19$} & \multicolumn{2}{|c|}{ Control $n=23$} \\
\hline Age (years) & \multicolumn{2}{|c|}{$62.9 \pm 10.0$} & \multicolumn{2}{|l|}{$63.0 \pm 6.4$} & \multicolumn{2}{|l|}{$62.8 \pm 8.6$} & \multicolumn{2}{|l|}{$58.2 \pm 8.6$} \\
\hline Males, n (\%) & \multicolumn{2}{|l|}{$3(25 \%)$} & \multicolumn{2}{|l|}{$3(42 \%)$} & \multicolumn{2}{|l|}{$6(32 \%)$} & \multicolumn{2}{|l|}{$8(35 \%)$} \\
\hline Height (m) & \multicolumn{2}{|c|}{$1.65 \pm 0.09$} & \multicolumn{2}{|l|}{$1.69 \pm 0.14$} & \multicolumn{2}{|l|}{$1.66 \pm 0.10$} & \multicolumn{2}{|c|}{$1.69 \pm 0.08$} \\
\hline Body mass (kg) & \multicolumn{2}{|c|}{$77.3 \pm 14.0$} & \multicolumn{2}{|l|}{$77.2 \pm 15.0$} & \multicolumn{2}{|l|}{$77.2 \pm 14.0$} & \multicolumn{2}{|c|}{$69.9 \pm 10.0$} \\
\hline Body mass index $\left(\mathrm{kg} / \mathrm{m}^{2}\right)$ & \multicolumn{2}{|l|}{$28.2 \pm 3.5$} & \multicolumn{2}{|l|}{$27.1 \pm 3.5$} & \multicolumn{2}{|l|}{$27.8 \pm 3.5^{*}$} & \multicolumn{2}{|l|}{$24.4 \pm 3.0$} \\
\hline \multirow[t]{2}{*}{ Harris Hip Score $(H H S)^{a b}$} & \multicolumn{2}{|c|}{$69.9 \pm 12.9$} & \multicolumn{2}{|l|}{$66.2 \pm 13.5$} & \multicolumn{2}{|l|}{$68.6 \pm 12.9$} & \multicolumn{2}{|l|}{$99.9 \pm 0.7$} \\
\hline & Affected & Contralateral & Most affected & Less affected & (Most) affected & Contralateral/Less affected & Left & Right \\
\hline Joint space width (mm) & $2.3 \pm 1.1$ & $3.5 \pm 0.7$ & $2.9 \pm 0.5$ & $3.0 \pm 0.5$ & $2.5 \pm 1.0^{*}$ & $3.3 \pm 0.7^{*}$ & $4.0 \pm 0.5$ & $3.9 \pm 0.6$ \\
\hline Kellgren-Lawrence grade & $\begin{array}{l}2, n=4 \\
3, n=8\end{array}$ & $\begin{array}{l}0, n=4 \\
1, n=8\end{array}$ & $\begin{array}{l}2, n=4 \\
3, n=3\end{array}$ & $\begin{array}{l}2, n=5 \\
3, n=2\end{array}$ & $\begin{array}{l}2, n=8 \\
3, n=11\end{array}$ & $\begin{array}{l}0, n=4 \\
1, n=8 \\
2, n=5 \\
3, n=2\end{array}$ & $\begin{array}{l}0, n=12 \\
1, n=11\end{array}$ & $\begin{array}{l}0, n=18 \\
1, n=5\end{array}$ \\
\hline
\end{tabular}

Values are mean (standard deviation) unless otherwise stated

$O A$ osteoarthritis

* $p<0.05$ hip OA compared to control group

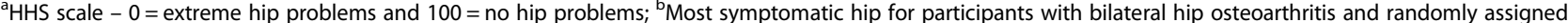
hip for control participants; Kellgren-Lawrence grading scale $-0=$ no radiographic features of hip osteoarthritis and $4=$ large osteophytes 
Table 2 Summary statistics for the effect of group (hip osteoarthritis versus control) on muscle strength and volume measures

\begin{tabular}{|c|c|c|c|c|c|c|}
\hline & Hip OA (mean \pm SD) & Control (mean $\pm S D)$ & $F, p$ & $\begin{array}{l}\text { Mean difference } \\
(\text { mean } \pm \text { SD) }\end{array}$ & $\begin{array}{l}95 \% \mathrm{Cl} \text { of mean } \\
\text { difference }\end{array}$ & Effect size \\
\hline \multicolumn{7}{|l|}{ Strength (Nm/kg) } \\
\hline Knee flexors & $0.977 \pm 0.292$ & $1.255 \pm 0.281$ & $9.579,0.004^{*}$ & $0.278 \pm 0.392$ & $0.096,0.460$ & 0.71 \\
\hline Knee extensors & $1.286 \pm 0.344$ & $1.664 \pm 0.328$ & $12.450,0.001^{*}$ & $0.378 \pm 0.462$ & $0.164,0.593$ & 0.82 \\
\hline Hip flexors & $0.898 \pm 0.331$ & $1.216 \pm 0.314$ & $9.866,0.003^{*}$ & $0.319 \pm 0.440$ & $0.113,0.524$ & 0.73 \\
\hline Hip extensors & $0.908 \pm 0.292$ & $1.216 \pm 0.281$ & $11.652,0.02^{*}$ & $0.307 \pm 0.392$ & $0.125,0.490$ & 0.78 \\
\hline Hip abductors & $0.662 \pm 0.209$ & $0.905 \pm 202$ & $14.34,0.001^{*}$ & $0.244 \pm 0.279$ & $0.113,0.374$ & 0.87 \\
\hline Hip adductors & $0.639 \pm 0.323$ & $0.834 \pm 0.314$ & $3.794,0.06$ & $0.194 \pm 0.436$ & $-0.008,0.397$ & 0.44 \\
\hline \multicolumn{7}{|l|}{ Volume $\left(\mathrm{cm}^{3} / \mathrm{kg}\right)$} \\
\hline TFL & $0.909 \pm 0.324$ & $0.816 \pm 0.300$ & $0.986,0.327$ & $0.094 \pm 0.410$ & $-0.285,0.098$ & 0.23 \\
\hline GMax & $9.560 \pm 2.336$ & $11.119 \pm 2.153$ & $5.268,0.028^{*}$ & $1.558 \pm 2.995$ & $0.182,2.934$ & 0.52 \\
\hline GMed & $4.031 \pm 0.722$ & $4.241 \pm 0.666$ & $1.001,0.324$ & $0.209 \pm 0.911$ & $-0.216,0.634$ & 0.23 \\
\hline GMin & $1.006 \pm 0.380$ & $1.525 \pm 0.352$ & $22.048,<0.001^{*}$ & $0.520 \pm 0.484$ & $0.295,0.744$ & 1.07 \\
\hline Add & $10.827 \pm 2.111$ & $12.489 \pm 1.947$ & $7.380,0.01^{*}$ & $1.662 \pm 2.668$ & $0.420,2.940$ & 0.62 \\
\hline Hams & $7.444 \pm 1.548$ & $9.117 \pm 1.426$ & $13.899,0.001^{*}$ & $1.673 \pm 1.957$ & $0.762,2.583$ & 0.85 \\
\hline Quad & $16.114 \pm 4.512$ & $20.769 \pm 4.160$ & $12.666,0.001^{*}$ & $4.655 \pm 5.701$ & $2.001,7.311$ & 0.82 \\
\hline
\end{tabular}

Add adductors, $\mathrm{Cl}$ confidence interval, Hams hamstrings, GMax gluteus maximus, GMed gluteus medius, GMin gluteus minimus, OA osteoarthritis, Quad quadriceps, TFL tensor fasciae latae

*Significant difference between groups $(p<0.05)$

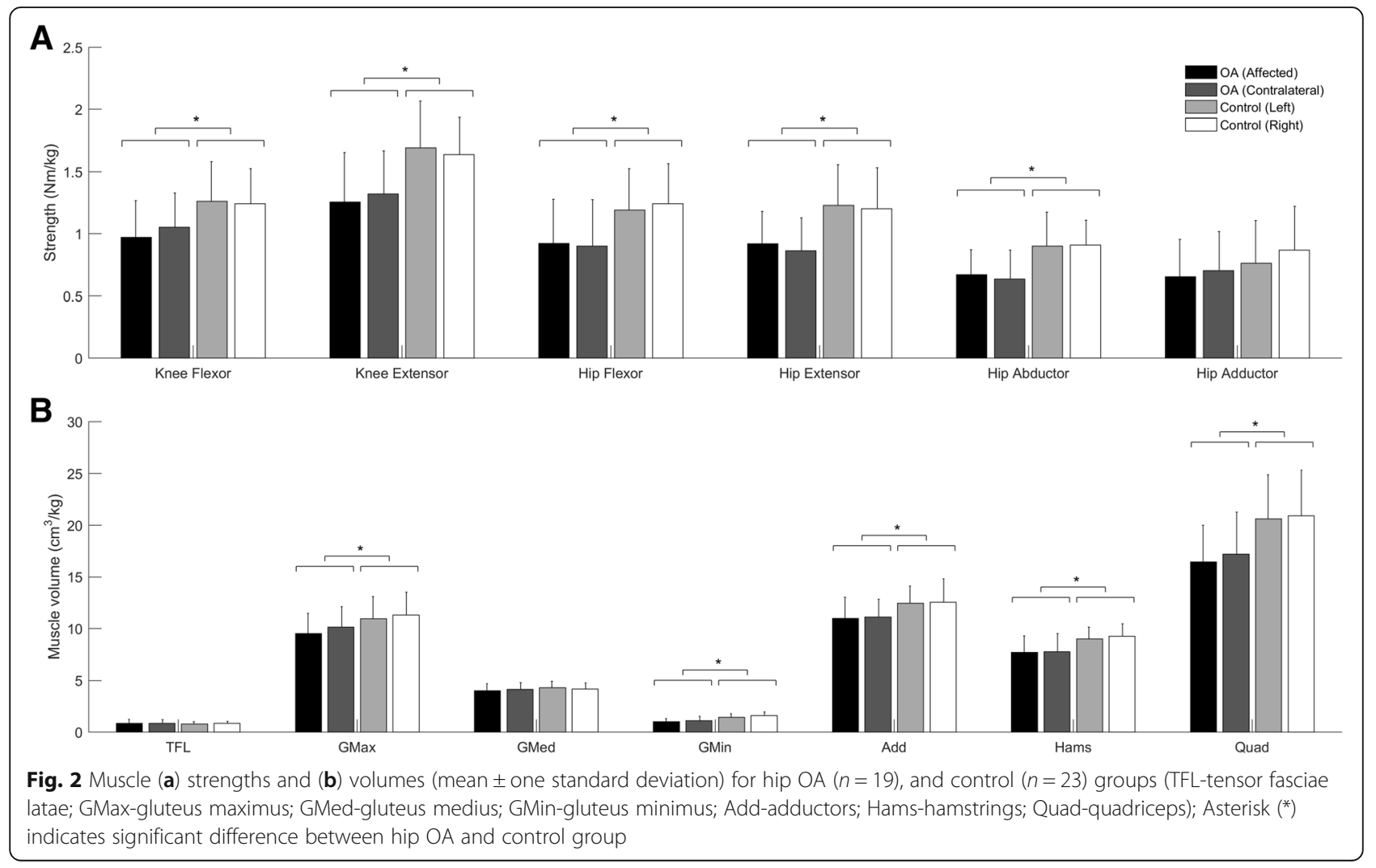


the healthy control group. Deficts in strength were detected for the hip flexors, extensors and abductors, and the knee flexors and extensors, but not the hip adductors. Smaller muscle volumes were detected for gluteus maximus, gluteus minimus, and the adductor, hamstring and quadricep muscle groups, but not for tensor fascia latae or gluteus medius. Previous research has demonstrated generalized lower limb muscle weakness and atrophy in advanced stages of hip OA [26], and in the hip abductors in earlier stages of the disease [27]. The main and novel finding of the present study was that pervasive deficits in lower limb muscle strength and size are also present in mild-to-moderate stages of the disease process. In contrast to our hypothesis, no between-limb differences in muscle strength or volume were found in our mild-to-moderate hip OA group. Between-limb asymmetries in muscle strength and volume instead appear to primarily be a feature of advanced stage hip OA [26].

\section{Muscle strength and volume in individuals with mild-to- moderate hip OA}

Individuals with hip OA exhibited strength deficits in the hip and knee flexors and extensors and hip abductors relative to control participants. Hip and knee muscle strength in the directions assessed was on average $22-26 \%$ lower than the control group. In general, the strength deficits in the hip OA group fall within the range reported (13-37\%) in previous investigations of hip muscle strength in hip OA $[12,39]$. Only hip adduction strength was not significantly lower in the hip OA group, but approached significance $(p=0.06)$ with an effect size of 0.44 , which may be clinically meaningful. We therefore interpret these findings to indicate that muscle weakness in the most affected limb in mild-to-moderate hip OA tends to be generalized rather than specific to individual muscles or muscle groups and that the magnitude of weakness is similar between mild-to-moderate and advanced hip OA. The underlying cause of muscle weakness in hip OA remains unclear but could arise from decreased physical activity and/or unloading of the lower extremity during physical activity [34], perhaps driven by some combination of pain and motor dysfunction. Unresolved questions that will require further investigation concern whether muscle weakness precedes or follows the onset of hip OA, and whether weakness is a contributing cause or consequence of hip OA.

Hip and knee muscle volumes were on average 5-30\% lower in individuals with hip OA across all muscle groups and individual muscles assessed, with the exception of tensor fascia latae and gluteus medius. The smaller muscle volumes in individuals with mild-to-moderate hip OA likely underpin their generalized deficits in hip and knee muscle strength, and coincide with reports of advanced hip OA [26]. In general, there was a correspondence in the amount of weakness detected at the joint level, and the atrophy of muscles that contributed to the measured strength. For example, the $22-26 \%$ lower strength of the knee flexors and extensors in the hip OA group, corresponded with $18-22 \%$ reductions in muscle volume of the hamstrings and quadriceps respectively, and suggest that muscle atrophy in hip OA is a major mechanism of underlying muscle weakness in these muscles. Our findings of lower gluteal (maximus and minimus) muscle volumes in individuals with hip OA compared to healthy controls are consistent with Zacharias et al. [27]. Further, our observations are broadly consistent with findings from a systematic review of muscle strength and size in hip OA relative to controls [26], which suggest that advanced unilateral hip OA is characterized by generalized muscle weakness and atrophy of muscles in the affected limb. Although gluteus medius had a 5\% lower volume in the hip OA group, this mean group difference was not statistically significant. The tensor fascia latae muscle volume was similarly not significantly different between groups. The absence of group differences in muscle volume for these muscles could be explained by possible group differences in hip abductor muscle activation capacity, force sharing between synergistic abductor muscles and muscle quality. A further possibility is that some muscles may compensate for reduction in strength of synergistic muscles as has been observed in individuals with knee muscle pathology following anterior cruciate ligament reconstruction [40]. Indeed Grimaldi et al. [20] reported larger volumes for gluteus medius compared to healthy controls in early stages of hip pathology compared to atrophy in later stages.

\section{Muscle strength and volume in the affected and less- affected/contralateral limbs of individuals with mild-to- moderate hip $O A$}

Lower muscle strength and volume did not differ significantly between-limbs in individuals with hip OA. Although 12 of $19(63 \%)$ of our cohort had unilateral hip OA (between-limb KL grade difference $\geq 1$ ), it is possible that the inclusion of 7 bilateral participants prevented asymmetries from being detected. However, a post hoc analysis of the unilateral hip OA sub-group did not reveal any clear trends to support strength or volume asymmetry (data not presented). Grimaldi et al. [20], who evaluated gluteal muscle size in individuals with mild and advanced unilateral hip OA, similarly observed no difference in muscle size between the affected and contralateral limb in the mild hip OA group. However, our observations contradict those of Zacharias et al. [27], who reported lower gluteal muscle volumes in individuals with moderate unilateral hip OA (KL grade 2: $n=7$; KL grade 3: $n=13$ ) compared to their contralateral side. When participants from Zacharias et al. 
[27] were dichotomized based on OA severity, only those with $\mathrm{KL}$ grade $=3$ demonstrated atrophy in the gluteal muscles. Our cohort was comprised of $42 \%$ of individuals with $\mathrm{KL}$ grade $=2$, which in light of the findings of Zacharias et al. [27], may suggest that muscle related asymmetry becomes more prominent with disease progression. A possible explanation for the lack of difference is muscle strength between limbs in hip OA is that rather than favouring the contralateral limb during the performance of functional tasks, individuals with mild-to-moderate hip OA unload both limbs through a reduction in overall physical activity.

Reduced muscle strength and volumes in the affected compared to contralateral limb are well documented in individuals with end-stage hip OA [14, 16, 18, 20-22]. In general, it is difficult to compare the findings from the present study to those from the literature due to differences in participant characteristics (single versus mixed sex, pre- versus post-total hip replacement), strength measurements (e.g. isometric versus isokinetic), and muscles assessed. However, findings from Zacharias et al. [27] and Grimaldi et al. [20], where a subset of lower limb muscle strength and/or muscle volumes were measured in participants with hip OA from across the disease spectrum using a consistent approach, suggest that asymmetries in strength and volume become more pronounced with disease progression. Interventions to retain bilateral muscle strength during early-middle stages of the disease therefore appear warranted in the management of hip OA. This recommendation is consistent with the evidence-based clinical practice guidelines for therapeutic exercise in the management of hip OA which recommend land-based therapeutic exercise, most notably strength training, to reduce pain, stiffness and self-reported disability, and improve physical function and range of motion [41].

\section{Strengths and limitations}

A strength of this study was that eligibility was based on radiographic and symptomatic criteria, which minimized the well-known risk of participant misclassification [42]. There were also several limitations to the study. First, the study was not sufficiently powered to perform a sub-group analysis of unilateral and bilateral participants. A future study with a larger sample size is required to more definitively determine whether strength and muscle volume asymmetry is evident within these hip OA sub-groups. More females were recruited to the hip OA and control groups than males (hip OA: 13 female, 6 male; control: 15 female, 8 male), which may be a source of experimental bias. While the hip OA group in our study had a significantly higher BMI than controls, strength and volume measures were normalised to body mass. We chose this method as it is common and therefore facilitates comparison of findings with other studies that have used the same approach and it also has physical meaning. Strength was assessed in the present study under isometric conditions, which may not reflect muscle function during dynamic conditions including activities of daily living. It was not possible to segment boundaries for some smaller muscles (e.g. internal/external hip rotators) or muscles with insertions outside the imaged segments (e.g. iliopsoas), and thus only large hip/knee spanning muscles and muscle groups were evaluated. Further, reliability of muscle segmentation from MRI scans was established using data from a single participant. It is important for future studies to more fully elucidate the implications of reduced muscle strength and volume in mild-to-moderate hip OA for motor function and disease progression. Multiple statistical comparisons were made in the present study, which has the potential to increase the risk of type 1 error. A statistical correction was not performed due of the exploratory nature of this study [43, 44]. It is noteworthy that the hip OA cohort from the present study also exhibited reduced self-selected walking speed and altered hip joint mechanics, including lower net hip joint loading over a reduced range of hip motion for a longer proportion of the gait cycle, when walking at their preferred gait speed relative to healthy control participants [34]. These findings are consistent with an underloading hypothesis for hip OA progression, perhaps due in part to muscle weakness, which could have implications for disease progression through altered mechano-biological processes within the joint [45].

\section{Conclusions}

The main conclusion from this study is that atrophic hip and knee muscle weakness is a distinct feature of mild-to-moderate hip OA. These strength and muscle size deficits tended to be generalized rather than localised to individual muscles and/or muscle groups in the lower limb, and have possible implications for daily function, quality of life and OA disease progression. While no evidence of between-limb asymmetry in muscle strength or volume was found in the present study, intervention early in the disease process to prevent the development of strength asymmetries that are characteristic of advanced hip OA appear warranted.

\section{Abbreviations \\ Add: Adductors; GMax: Gluteus maximus; GMed: Gluteus medius; \\ GMin: Gluteus minimus; Hams: Hamstrings; HHS: Harris hip score; ICC: Intra- class correlation coefficient; JSW: Joint space width; KL: Kellgren-Lawrence; MRI: Magnetic resonance imaging; OA: Osteoarthritis; Quad: Quadricpes; TFL: Tensor fasciae latae}

Acknowledgements

The authors wish to thank Dr. Gary Shepherd (Qscan Radiology Clinics), Dr. Peter Mills, and the participants for support with the project. 


\section{Funding}

Funding was provided by a Griffith University Area of Strategic Investment Grant in Chronic Disease Prevention for participant anterior-posterior radiographs of the pelvis and hips. AL received a Griffith University Postgraduate Research Scholarship and a Griffith University International Postgraduate Research Scholarship.

\section{Availability of data and materials}

The datasets used and/or analyzed during the current study are available from the corresponding author on reasonable request.

\section{Authors' contributions}

$A L, M C, B B$ and $R B$ conceived the design of this study. $A L$ and $M C$ acquired the data. $L D, A L, R B$ carried out the analysis and interpretation of the data. $L D, A L$ and $R B$ drafted the article. All authors revised the manuscript for intellectual content and approved the final version.

\section{Ethics approval and consent to participate}

Ethical approval was obtained from Griffith Univeristy Human Research Ethics Committee and all participants provided written informed consent.

\section{Consent for publication}

Not applicable.

\section{Competing interests}

Laura Diamond is an editorial board member for BMC Musculoskeletal Disorders. The other authors declare that they have no competing interests.

\section{Publisher's Note}

Springer Nature remains neutral with regard to jurisdictional claims in published maps and institutional affiliations.

\section{Author details}

${ }^{1}$ Menzies Health Institute Queensland, School of Allied Health Sciences, Griffith University, Gold Coast, QLD 4222, Australia. ${ }^{2}$ Pontifical Catholic University (PUCRS), Porto Alegre, Brazil. ${ }^{3}$ University of Rio dos Sinos (UNISINOS), São Leopoldo, Brazil. ${ }^{4}$ Australian Catholic University, Brisbane, QLD 4014, Australia. ${ }^{5}$ Centre of Clinical Research Excellence in Spinal Pain Injury \& Health, School of Health \& Rehabilitation Sciences, The University of Queensland, Brisbane, QLD, Australia.

Received: 28 March 2018 Accepted: 14 August 2018

Published online: 21 August 2018

\section{References}

1. DiBonaventura M, Gupta S, McDonald M, Sadosky A. Evaluating the health and economic impact of osteoarthritis pain in the workforce: results from the National Health and wellness survey. BMC Musculoskelet Disord. 2011;12:83.

2. Lane NE. Osteoarthritis of the hip. N Engl J Med. 2007:357:1413-21.

3. Eitzen I, Fernandes L, Kallerud H, Nordsletten L, Knarr B, Risberg MA. Gait characteristics, symptoms, and function in persons with hip osteoarthritis: a longitudinal study with 6 to 7 years of follow-up. J Orthop Sports Phys Ther. 2015;45:539-49.

4. Constantinou M, Barrett R, Brown M, Mills P. Spatial-temporal gait characteristics in individuals with hip osteoarthritis: a systematic literature review and meta-analysis. J Orthop Sports Phys Ther. 2014;44:291-7.

5. Castaño-Betancourt MC, Rivadeneira F, Bierma-Zeinstra S, Kerkhof HJM Hofman A, Uitterlinden AG, Van Meurs JBJ. Bone parameters across differen types of hip osteoarthritis and their relationship to osteoporotic fracture risk. Arthritis Rheum. 2013;65:693-700.

6. Arnold CM, Faulkner RA. The effect of aquatic exercise and education on lowering fall risk in older adults with hip osteoarthritis. J Aging Phys Act. 2010:18:245-60.

7. Bennell KL, Hinman R. Exercise as a treatment for osteoarthritis. Curr Opin Rheumatol. 2005:17:634-40.

8. Lane NE, Buckwalter JA. Exercise and osteoarthritis. Curr Opin Rheumatol. 1999:11:413-6.

9. McNair PJ, Simmonds MA, Boocock MG, Larmer PJ. Exercise therapy for the management of osteoarthritis of the hip joint: a systematic review. Arthritis Res Ther. 2009;11:1-9.
10. Puett DW, Griffin MR. Published trials of nonmedicinal and noninvasive therapies for hip and knee osteoarthritis. Ann Intern Med. 1994;121:133-40.

11. Tilden HM, Reicherter $A E$, Reicherter F. Use of an aquatics program for older adults with osteoarthritis from clinic to the community. Top Geriatr Rehabil. 2010;26:128-39.

12. Arokoski MH, Arokoski JP, Haara M, Kankaanpaa M, Vesterinen M, Niemitukia $\mathrm{LH}$, Helminen HJ. Hip muscle strength and muscle cross sectional area in men with and without hip osteoarthritis. J Rheumatol. 2002;29:2185-95.

13. Madsen OR, Brot C, Petersen MM, Sorensen OH. Body composition and muscle strength in women scheduled for a knee or hip replacement. A comparative study of two groups of osteoarthritic women. J Clin Rheumatol. 1997;16:39-44.

14. Rasch A, Bystrom AH, Dalen N, Berg HE. Reduced muscle radiological density, cross-sectional area, and strength of major hip and knee muscles in 22 patients with hip osteoarthritis. Acta Orthop. 2007:78:505-10.

15. Rasch A, Dalen N, Berg HE. Muscle strength, gait, and balance in 20 patients with hip osteoarthritis followed for 2 years after THA. Acta Orthop. 2010;81:183-8.

16. Reardon K, Galea M, Dennett X, Choong P, Byrne E. Quadriceps muscle wasting persists 5 months after total hip arthroplasty for osteoarthritis of the hip: a pilot study. Intern Med J. 2001:31:7-14.

17. Rossi MD, Brown LE, Whitehurst MA. Assessment of hip extensor and flexor strength two months after unilateral total hip arthroplasty. J Strength Cond Res. 2006;20:262-7.

18. Suetta C, Aagaard P, Rosted A, Jakobsen AK, Duus B, Kjaer M, Magnusson SP. Training-induced changes in muscle CSA, muscle strength, EMG, and rate of force development in elderly subjects after long-term unilateral disuse. J Appl Physiol. 2004;97:1954-61.

19. Suetta C, Andersen JL, Dalgas U, Berget J, Koskinen S, Aagaard P, Magnusson SP, Kjaer M. Resistance training induces qualitative changes in muscle morphology, muscle architecture, and muscle function in elderly postoperative patients. J Appl Physiol. 2008;105:180-6.

20. Grimaldi A, Richardson C, Durbridge G, Donnelly W, Darnell R, Hides J. The association between degenerative hip joint pathology and size of the gluteus maximus and tensor fascia lata muscles. Man Ther. 2009;14:611-7.

21. Rasch A, Bystrom AH, Dalen N, Martinez-Carranza N, Berg HE. Persisting muscle atrophy two years after replacement of the hip. J Bone Joint Surg. 2009;91:583-8

22. Suetta C, Aagaard P, Magnusson SP, Andersen LL, Sipila S, Rosted A, Jakobsen AK, Duus B, Kjaer M. Muscle size, neuromuscular activation, and rapid force characteristics in elderly men and women: effects of unilateral long-term disuse due to hip-osteoarthritis. J Appl Physiol. 2007;102:942-8.

23. Zhang W, Nuki G, Moskowitz RW, Abramson S, Altman RD, Arden NK, Bierma-Zeinstra S, Brandt KD, Croft P, Doherty M, et al. OARSI recommendations for the management of hip and knee osteoarthritis: part III: changes in evidence following systematic cumulative update of research published through January 2009. Osteoarthr Cartil. 2010;18:476-99.

24. Svege I, Fernandes L, Nordsletten L, Holm I, Risberg MA. Long-term effect of exercise therapy and patient education on impairments and activity limitations in people with hip osteoarthritis: secondary outcome analysis of a randomized clinical trial. Phys Ther. 2016:96:818-27.

25. French HP, Cusack T, Brennan A, Caffrey A, Conroy R, Cuddy V, FitzGerald OM, Fitzpatrick M, Gilsenan C, Kane D, et al. Exercise and manual physiotherapy arthritis research trial (EMPART) for osteoarthritis of the hip: a multicente randomized controlled trial. Arch Phys Med Rehabil. 2013;94:302-14.

26. Loureiro A, Mills PM, Barrett RS. Muscle weakness in hip osteoarthritis: a systematic review. Arthritis Care Res. 2013;65:340-52

27. Zacharias A, Pizzari T, English DJ, Kapakoulakis T, Green RA. Hip abductor muscle volume in hip osteoarthritis and matched controls. Osteoarthr Cartil. 2016;24:1727-35.

28. Grimaldi A, Richardson C, Stanton W, Durbridge G, Donnelly W, Hides J. The association between degenerative hip joint pathology and size of the gluteus medius, gluteus minimus and piriformis muscles. Man Ther. 2009;14:605-10.

29. Roos EM, Herzog W, Block JA, Bennell KL. Muscle weakness, afferent sensory dysfunction and exercise in knee osteoarthritis. Nat Rev Rheumatol. 2011;7:57-63.

30. Mahomed NN, Arndt DC, McGrory BJ, Harris WH. The Harris hip score: comparison of patient self-report with surgeon assessment. J Arthroplast. 2001;16:575-80

31. Kellgren JH, Lawrence JS. Radiological assessment of rheumatoid arthritis. Ann Rheum Dis. 1957;16:485-93. 
32. Auleley GR, Giraudeau B, Dougados M, Ravaud P. Radiographic assessment of hip osteoarthritis progression: impact of reading procedures for longitudinal studies. Ann Rheum Dis. 2000;59:422-7.

33. Altman RD, Gold GE. Atlas of individual radiographic features in osteoarthritis, revised. Osteoarthr Cartil. 2007;15(Suppl A):A1-56.

34. Constantinou M, Loureiro A, Carty C, Mills P, Barrett R. Hip joint mechanics during walking in individuals with mild-to-moderate hip osteoarthritis. Gait Posture. 2017;53:162-7.

35. Von Elm E, Altman DG, Egger M, Pocock SJ, Gøtzsche PC, Vandenbroucke $J P$. The strengthening the reporting of observational studies in epidemiology (STROBE) statement: guidelines for reporting observational studies. Prev Med. 2007;45:247-51.

36. Carty CP, Barrett RS, Cronin NJ, Lichtwark GA, Mills PM. Lower limb muscle weakness predicts use of a multiple- versus single-step strategy to recover from forward loss of balance in older adults. J Gerontol A Biol Sci Med Sci. 2012;67:1246-52

37. Kellis $\mathrm{E}$, Baltzopoulos V. Gravitational moment correction in isokinetic dynamometry using anthropometric data. Med Sci Sports Exerc. 1996; 28:900-7.

38. Dempster WT. Space requirements of the seated operator: geometrical, kinematic, and mechanical aspects of the body, with special reference to the limbs. 1955.

39. Klausmeier V, Lugade V, Jewett BA, Collis DK, Chou LS. Is there faster recovery with an anterior or anterolateral THA? A pilot study. Clin Orthop Relat Res. 2010;468:533-41.

40. Konrath JM, Vertullo CJ, Kennedy BA, Bush HS, Barrett RS, Lloyd DG. Morphologic characteristics and strength of the hamstring muscles remain altered at 2 years after use of a hamstring tendon graft in anterior cruciate ligament reconstruction. Am J Sports Med. 2016;44:2589-98.

41. Brosseau L, Wells GA, Pugh AG, Smith CAM, Rahman P, Gallardo ICA Toupin-April K, Loew L, De Angelis G, Cavallo S. Ottawa panel evidencebased clinical practice guidelines for therapeutic exercise in the management of hip osteoarthritis. Clin Rehabil. 2016;30:935-46.

42. Kim C, Linsenmeyer KD, Vlad SC, Guermazi A, Clancy MM, Niu J, Felson DT. Prevalence of radiographic and symptomatic hip osteoarthritis in an urban United States community: the Framingham osteoarthritis study. Arthritis Rheumatol. 2014;66:3013-7.

43. Bender R, Lange S. Adjusting for multiple testing--when and how? J Clin Epidemiol. 2001;54:343-9.

44. Perneger TV. What's wrong with Bonferroni adjustments. BMJ. 1998;316: $1236-8$.

45. Saxby DJ, Lloyd DG. Osteoarthritis year in review 2016: mechanics. Osteoarthr Cartil. 2017:25:190-8.

Ready to submit your research? Choose BMC and benefit from:

- fast, convenient online submission

- thorough peer review by experienced researchers in your field

- rapid publication on acceptance

- support for research data, including large and complex data types

- gold Open Access which fosters wider collaboration and increased citations

- maximum visibility for your research: over $100 \mathrm{M}$ website views per year

At $\mathrm{BMC}$, research is always in progress.

Learn more biomedcentral.com/submissions 